\title{
The characterisation of subjective cognitive decline
}

\author{
FrankJessen, Rebecca E Amariglio, Rachel F Buckley, Wiesje M van der Flier, Ying Han, José Luis Molinuevo, Laura Rabin, Dorene M Rentz, \\ Octavio Rodriguez-Gomez, Andrew J Saykin, Sietske A M Sikkes, Colette M Smart, Steffen Wolfsgruber, Michael Wagner
}

\begin{abstract}
A growing awareness about brain health and Alzheimer's disease in the general population is leading to an increasing number of cognitively unimpaired individuals, who are concerned that they have reduced cognitive function, to approach the medical system for help. The term subjective cognitive decline (SCD) was conceived in 2014 to describe this condition. Epidemiological data provide evidence that the risk for mild cognitive impairment and dementia is increased in individuals with SCD. However, the majority of individuals with SCD will not show progressive cognitive decline. An individually tailored diagnostic process might be reasonable to identify or exclude underlying medical conditions in an individual with SCD who actively seeks medical help. An increasing number of studies are investigating the link between SCD and the very early stages of Alzheimer's disease and other neurodegenerative diseases.
\end{abstract}

\section{Introduction}

With an ageing population, concerns about cognitive decline are becoming an increasingly relevant topic that arises during medical consultations. Concerns about cognitive decline can be associated with different objective levels of cognitive and functional impairment, which are revealed by clinical and neuropsychological examinations. Dementia is the most severe level of impairment and is defined by cognitive deficits that impair daily functioning and lead to loss of independence. ${ }^{1,2}$ Similar to dementia, mild cognitive impairment (MCI) is also characterised by objective cognitive impairment. However, by contrast with dementia, the day-to-day functioning of individuals with MCI remains largely intact and independence is preserved. ${ }^{3}$ Some individuals experience a subjective decrease in cognitive function, but cognitive performance by neuropsychological testing and in daily functioning shows no evidence of objective cognitive impairment. In clinical practice, these individuals are generally considered healthy. Regardless of the absence of evidence for objective cognitive impairment, the subjective decline in cognitive function experienced by individuals might become increasingly important for clinicians, because the number of individuals with such concerns who seek medical help and advice is growing. In 2014, the term subjective cognitive decline (SCD) was conceived by researchers to describe this condition, which has received increasing attention because of evidence of its association with an increased risk of future objective cognitive decline. ${ }^{46}$

One aim of this Personal View is to propose diagnostic and counselling considerations to health-care providers for individuals who seek medical help for SCD. Importantly, we do not propose actively searching or screening for individuals with SCD within or outside the clinical setting. A second aim is to provide information about the research findings and priorities for SCD to date, with a particular focus on studies looking at associations between SCD and preclinical Alzheimer's disease within current research frameworks.

\section{Definition and characterisation of SCD}

The association between subjective decline in cognitive functioning and ageing, objective performance, and the future risk of cognitive decline has been addressed in diverse concepts and in various studies since the 1980s. In 2014, an international working group of researchers and clinicians, known as the SCD-initiative (SCD-I), proposed a common framework for SCD research that provided standardised terminology and criteria. ${ }^{4}$ The proposed SCD criteria were developed within the context of Alzheimer's disease research; however, we believe that the same SCD criteria can also be useful in the health-care setting. The SCD criteria include two major features. First, a selfexperienced persistent decline in cognitive capacity, compared with a previously normal cognitive status, which is unrelated to an acute event. This criterion infers that SCD reflects a state of cognitive decline from the perspective of the individual; observation of such a decline by others is not required. The second criterion is normal performance on standardised cognitive tests used to classify MCI, adjusted for age, sex, and education. As such, the individual's cognition is unimpaired from an objective standpoint. Conditions that are defined by objective cognitive impairment, such as MCI or dementia, are distinct from SCD. Of note, SCD is not a diagnostic category of the International Statistical Classification of Diseases-10, the International Classification of Diseases-11, and the Diagnostic and Statistical Manual of Mental Disorders-5.

SCD is a broad condition that develops differently over time as a result of the variety of potential underlying causes (figure 1). To identify the potential causes of SCD in an individual, we propose that the concern of cognitive decline is evaluated by obtaining the following information from them: (1) which cognitive domains are affected (eg, memory, language, executive functioning, visual-spatial abilities, orientation, and attention); (2) whether specific worries are associated with the cognitive decline; (3) when the onset of cognitive decline occurred; (4) whether there is an association with any physical or mental conditions; and (5) whether there is an association with the use of medication, alcohol, or other substances.

To characterise the concern of cognitive decline further, particularly with regards to severity, several additional tools are available. ${ }^{8}$ Examples include the Cognitive Function Index, ${ }^{9}$ the Cognitive Change Index, ${ }^{10}$ the Everyday Cognition questionnaire, ${ }^{11}$ the Subjective Cognitive Decline
Lancet Neurol 2020 Published Online January 17, 2020 https://doi.org/10.1016/ S1474-4422(19)30368-0 See Online/Comment https://doi.org/10.1016/ S1474-4422(20)30002-8 Department of Psychiatry, Medical Faculty (Prof F Jessen MD) and Excellence Cluster on Cellular Stress Responses in Aging-Associated Diseases (CECAD) (Prof F Jessen), University of Cologne, Cologne, Germany; German Center for Neurodegenerative Diseases (DZNE), Bonn, Germany (ProfFJessen, SWolfsgruber $\mathrm{PhD}$ M Wagner PhD); Center for Alzheimer Research and Treatment (RE Amariglio PhD, D M Rentz PsyD), Department of Neurology (R F Buckley PhD, SA M Sikkes PhD), Brigham and Women's Hospital and Department of Neurology Massachusetts General Hospital, Harvard Medical School, Boston, MA, USA Florey Institute (R F Buckley) and Melbourne School of Psychological Sciences, University of Melbourne, Parkville, VIC, Australia (R F Buckley); Alzheimer Center Amsterdam, Department of Neurology, Amsterdam Neuroscience

(W M van der Flier PhD, S A M Sikkes) and Department of Epidemiology and Biostatistics (W M van der Flier S A M Sikkes), Vrije Universiteit Amsterdam, Amsterdam UMC Amsterdam, Netherlands; Department of Neurology, Xuanwu Hospital of Capital Medical University, Beijing, China (Y Han MD); Center of Alzheimer's Disease, Beijing Institute for Brain Disorders, Beijing, China (Y Han); Beijing Institute of Geriatrics, Beijing, China (Y Han); National Clinical Research Center for Geriatric Disorders, Beijing, China (Y Han); Alzheimer's Disease and Other Cognitive Disorders Unit, Hospital Clínic, Barcelona, Spain 


\section{(J L Molinuevo MD); Barcelonaßeta Brain Research Center, Pasqual Maragall Foundation, Barcelona, Spain (J L Molinuevo); Department of Psychology, Brooklyn College and The Graduate Center of CUNY, Brooklyn, NY, USA (L Rabin, PhD); Department of Neurology, Albert Einstein College of Medicine, New York, NY, USA (L Rabin); Alzheimer Research Center and Memory Clinic of Fundació ACE, Institut Català de Neurociències Aplicades, Barcelona, Spain \\ (O Rodriguez-Gomez MD); Department of Radiology and Imaging Sciences \\ (A) Saykin PsyD), Indiana Alzheimer Disease Center (A) Saykin), and Department of Medical and Molecular Genetics (A) Saykin), Indiana University School of Medicine, Indianapolis, IN, USA; Department of Psychology (C M Smart PhD) and Institute on Aging \& Lifelong Health, University of Victoria, \\ Victoria, BC, Canada \\ (CM Smart); and Department \\ of Neurodegeneration and Geriatric Psychiatry, University Hospital Bonn, Bonn, Germany (M Wagner) \\ Correspondence to: Prof Frank Jessen, Department of Psychiatry, University of Cologne, \\ Cologne, 50924, Germany frank.jessen@uk-koeln.de}

$$
\begin{aligned}
& \text { A) Reversible SCD } \\
& \text { No objective cognitive decline to a level of impairment }
\end{aligned}
$$

B) Stable, non-reversible SCD

No objective cognitive decline to a level of impairment

C) SCD with subsequent progressive cognitive decline to impairment or dementia

Figure 1: Schematic representation of trajectories of SCD and objective cognitive function over time

(A) SCD occurs but remits fully, and objective cognitive functions remain stable. Conditions underlying SCD include depression, side-effects from medication, or intermittent sleep disturbances. (B) SCD occurs and continues without remitting (continuous green colour); objective cognitive functions remain largely stable. The normal ageing process can be an underlying cause of this type of SCD. (C) SCD occurs and objective cognitive functions subsequently deteriorates to dementia. This deterioration can be caused by neurodegenerative disease, including, but not limited to, Alzheimer's disease. The proportion of individuals in each of the three SCD trajectories has not yet been fully delineated; however, the majority of individuals with SCD will not decline to dementia. The colour scheme indicates an absence of SCD (white), SCD (green), and objective impairment or dementia (red). SCD=subjective cognitive decline.

questionnaire, $^{12}$ the SCD interview, ${ }^{13}$ or a simple twoquestion approach about their decline in memory and any associated concerns. ${ }^{14}$ No single gold standard instrument or sufficiently validated cutoff on any scale can differentiate individuals with SCD from those without SCD in the clinical setting, where SCD is currently defined by use of the two basic SCD-I criteria. ${ }^{4}$ A validated cutoff would be useful for classifying specific groups of individuals and for quantifying the severity of SCD in a research setting. As such, research priorities of the SCD-I are to harmonise existing measures and create a validated SCD scale. ${ }^{8}$

\section{Discrimination of SCD from $\mathrm{MCl}$}

The absence of objective cognitive impairment distinguishes SCD from MCI. In principle, MCI was developed as a diagnostic entity, whereby the absence or presence of cognitive impairment is decided on the basis of clinical judgment. Cognitive test performance of the patient can support this diagnostic judgement. ${ }^{3}$ When clinical judgement is used alone, there may be a diagnostic overlap of SCD and MCI between clinicians However, in many clinical settings, the distinction between SCD and MCI is mainly based on either short or extended psychometric tests. ${ }^{3,15}$ When differentiating MCI (impaired objective performance) from SCD (unimpaired objective performance) on the basis of psychometric tests, we consider short cognitive screening tests, such as the Mini-Mental State Examination or the Montreal Cognitive Assessment, to have limited diagnostic accuracy. ${ }^{16}$ Instead, we suggest comprehensive neuropsychological test batteries that assess multiple cognitive domains, and for which ageadjusted, sex-adjusted, and education-adjusted normative data are available. ${ }^{17}$ No universally accepted single cutoff exists to define cognitive impairment for the purpose of differentiating between SCD and MCI. Commonly proposed approaches, however, include standard deviation (SD)-based cutoffs (ie, scores of $>1.5 \mathrm{SD}$ below the normative mean on any test within a specific cognitive domain; scores of $>1.0$ SD below the normative mean on at least two separate tests of one cognitive domain; or a score of $>1.0$ SD below the normative mean in at least three cognitive domains). ${ }^{18}$ To differentiate between SCD and MCI, individuals with SCD would need to score higher than the cutoffs, whereas individuals with MCI or dementia would need to score below the cutoffs. Alternatively, a clinical decision that is based on all the available clinical and neuropsychological information might be used to determine the extent of cognitive impairment. ${ }^{19,20}$

Of particular note, individuals with SCD and cognitively unimpaired individuals without SCD are only distinguished by reported feelings of subjective cognitive decline, which are experienced by individuals with SCD and not in individuals without SCD. The distinction is not made by cognitive testing, because individuals with SCD and cognitively unimpaired individuals without SCD are, by definition, objectively unimpaired, and they both perform above the cutoff for impairment in cognitive tests.

\section{Prevalence of SCD in an aging population}

Multiple physiological and subthreshold disease-related mechanisms contribute to the decline in cognitive function with aging. Typically, such declines in cognitive function involve the cognitive domains of processing speed, executive functions, memory, and visuospatial abilities. ${ }^{21,22}$ Most individuals notice some cognitive changes with increasing age. Population-based studies ${ }^{14,23}$ suggest that between $50 \%$ and $80 \%$ of older individuals (aged 70 years and older) who perform within normal ranges on cognitive tests, report some form of perceived decline in cognitive functioning when asked.

\section{$S C D$ and risk of cognitive decline}

A meta-analysis ${ }^{5}$ of longitudinal epidemiological studies of cognitively unimpaired individuals with SCD (with at least 4 years of follow-up data) found a future decline to dementia in $14 \%$ of individuals and a future decline to $\mathrm{MCI}$ in $27 \%$ of individuals. These estimates are limited by the inclusion of all age groups in the analysis. ${ }^{5}$ Despite this limitation and even though SCD is not related to progressive cognitive deterioration in most individuals, SCD could be an early indicator of future cognitive decline for some individuals. ${ }^{6}$ Long-term prospective studies ${ }^{24,25}$ in individuals who eventually went on to develop dementia, suggest that SCD occurs, on average, around 10 years before the dementia diagnosis.

The SCD criteria publication ${ }^{4}$ describes several characteristics of SCD, for which there is evidence that they indicate a particular risk of objective cognitive decline. These characteristics are known as SCD plus features (panel) $)^{4}$, which are subject to ongoing validation and refinement, and they might be modified in the future. 
The first SCD plus feature is the subjective decline in the memory of an individual irrespective of decline in other domains. Of note, this SCD plus feature is based on the fact that most SCD studies have focused on reports of memory. ${ }^{14}$ The association between the extent of subjective decline in other cognitive domains and future objective cognitive decline is uncertain. ${ }^{33,34}$ The second SCD plus characteristic is the onset of SCD within the past 5 years. This feature is based on longitudinal studies ${ }^{24,25}$ showing that the onset of SCD occurs approximately 10 years before a dementia diagnosis. Considering that individuals with cognitive decline will first progress to MCI before dementia, onset of SCD of more than 5 years is less likely to be related to future dementia than an onset of SCD within 5 years. The third characteristic of SCD plus is the onset of SCD in an individual over the age of 60 years. In individuals younger than 60 years of age, the likelihood of a medical condition causing future cognitive decline and dementia is low, which suggests that the likelihood of SCD in individuals younger than 60 years being related to other or potentially reversible causes (eg, depression) is higher than in individuals aged 60 years or older. The fourth SCD plus characteristic is the expression of concerns (worries) associated with SCD. There is evidence that individuals who express concerns about the perceived decline in cognitive function have an increased risk of developing objective cognitive decline or dementia in the future. ${ }^{14,26}$ The final SCD plus characteristic is the confirmation of cognitive decline by an observer. For an individual with SCD, there is evidence that a reported decline in cognition by an observer is associated with an increased likelihood of future cognitive decline. ${ }^{30}$ There is also evidence to suggest that only the individual with SCD will experience cognitive decline at the very early stages, whereas observers will notice mild cognitive dysfunction at a slightly more advanced stage of cognitive decline. This sequence has been reported to occur even before individuals reach the stage of MCI. ${ }^{31,32}$

We propose two additional SCD plus features that were not part of the original SCD plus criteria. ${ }^{4}$ The first is consistent SCD over time (as opposed to SCD on sporadic occasions or over limited time periods). There is evidence that individuals who consistently and repeatedly report a subjective decline in cognitive function over time are at a greater risk of future objective cognitive decline than those who report a subjective decline in cognitive function on only one occasion. . $3,27,28$ The second proposed SCD plus feature is seeking medical help because of $\mathrm{SCD}$. This feature is associated with a higher risk of future objective cognitive decline in individuals with SCD than in those with SCD who do not seek medical help. ${ }^{6,29}$

\section{Differential diagnosis and counselling}

Experiencing cognitive decline can prompt some individuals to seek medical advice. Access to memory services varies between health-care systems and depends on
Panel: Features that increase the risk of cognitive decline

(SCD plus)

- Subjective decline in memory irrespective of function in other cognitive domains 5.14

- Onset of SCD within the past 5 years ${ }^{24,25}$

- Onset of SCD at 60 years and older ${ }^{4}$

- Concern (worry) associated with SCD ${ }^{14,26}$

- Persistence of SCD over time ${ }^{23,27,28 *}$

- Seeking of medical help ${ }^{6,29 *}$

- Confirmation of cognitive decline by an observer $r^{30,31,32}$

*Not part of the original SCD plus features. ${ }^{4} \mathrm{SCD}=$ subjective cognitive decline

cultural factors within and across countries. ${ }^{35,36}$ Two memory clinics in Europe reported that $25-40 \%$ of individuals who sought help for cognitive decline fulfilled the criteria for SCD..$^{20,37}$ We propose that an individually tailored diagnostic process might be reasonable to identify or exclude underlying medical conditions in an individual with SCD who actively seeks medical help. Common brain diseases, including neurodegenerative diseases, such as Alzheimer's disease, Parkinson's disease, cerebrovascular disease, inflammatory brain diseases, and head trauma, can cause a subjective decline in cognitive functioning. Most psychiatric disorders and subclinical psychiatric conditions can affect cognition; the most common of which are depression, anxiety, and sleep disorders. Other medical conditions related to cognition include metabolic diseases (eg, diabetes), endocrine diseases (eg, thyroid dysfunction), hypertension, heart disease, anaemia, liver diseases, kidney diseases, infectious diseases, and nutritional deficiencies. Additionally, substance misuse and several drugs can affect cognition (eg, sedatives, anticholinergics, opioids, and corticosteroids). Somatoform disorders, sensitive self-monitoring, personality traits (eg, neuroticism), and a fear of dementia can also trigger concerns about cognitive decline or can strongly enhance the experience of normal age-related cognitive changes..$^{38}$ Finally, for many individuals with SCD, it is likely that this condition is related to the normal physiological ageing process. Of note, SCD is often associated with mild symptoms of depression or anxiety, ${ }^{39}$ but we suggest that these symptoms are not prematurely considered to be the cause of SCD, as they might co-occur with SCD because of a common underlying cause, or as a result of SCD itself.

We propose that the extent of the diagnostic evaluation, beyond a descriptive clinical and potentially neuropsychological evaluation, in a person with SCD who presents to the physician with a request for medical help, will depend on the individual situation and the respective shared decision process. In individuals for whom a specific underlying medical or psychiatric condition is identified, we propose that the association between SCD and the underlying condition should be discussed, in addition to the potential consequences, treatment options, 
and prognoses. If no specific cause of SCD is identified, we suggest explaining to the individual that SCD is associated with a mildly increased risk of future cognitive decline, particularly in those with so called SCD plus features. ${ }^{4}$ We also propose explaining that SCD can resolve spontaneously, and that most individuals with SCD will not experience objective cognitive decline in the near future (figure 1). ${ }^{5,6}$ Clinical follow-up might be considered on the basis of the individual's decision. We also propose that individuals with SCD should be advised on strategies to support brain health. On the basis of the epidemiological evidence on the modifiable risk factors for dementia, ${ }^{40}$ we suggest that this advice should include control of hypertension and diabetes, treatment of mood disorders, physical exercise, weight control, a Mediterranean-style diet, smoking cessation, cognitive and social engagement activities, high-quality sleep, stress reduction, and the use of hearing aids (if needed). We propose that such advice should be adjusted to the individual's characteristics, needs, and preferences, and that it is supported by technical aids, such as internet-based tools for self-guided brain-health activities. ${ }^{41}$

\section{SCD and the Alzheimer's disease research framework}

There are two currently proposed sets of research criteria for Alzheimer's disease. The first set was published by the International Working Group (IWG) in 2014. ${ }^{42}$ The IWG defines Alzheimer's disease as a clinicopathological entity that is classified by the presence of amyloid $\beta$ and tau protein pathology, and specific clinical symptoms, such as objective memory recall deficit (not compensated by cueing) with or without impairment of daily functioning. As such, individuals with Alzheimer's disease can present with prodromal symptomatology or with full dementia. ${ }^{42}$ Alzheimer's disease pathology is identified by biomarkers. The respective biomarkers proposed by the IWG framework for amyloid $\beta$ pathology include the concentration of amyloid $\beta$ peptide $1-42$ in the CSF or presence of amyloid $\beta$ by PET scan. Tau pathology was proposed to be indicated by total or phosphorylated tau protein in the CSF. ${ }^{42}$ In 2016, a combined working group, consisting of the IWG and the US Alzheimer's Association (AA), proposed a definition and criteria for preclinical Alzheimer's disease, which describes the stage at which Alzheimer's disease pathology is present, but symptoms have not yet developed. ${ }^{43}$

The term preclinical Alzheimer's disease was already proposed by the National Institute of Aging-Alzheimer's Association (NIA-AA) in 2011. ${ }^{44}$ According to the IWG-AA criteria from 2016, preclinical Alzheimer's disease is defined by the presence of amyloid $\beta$ and tau pathology in individuals without objectively impaired cognition. By contrast, the presence of either amyloid $\beta$ pathology or tau pathology is considered to indicate the asymptomatic stage, where individuals are at risk of developing Alzheimer's disease. ${ }^{43}$ The NIA-AA have already acknowledged that subtle cognitive decline occurs in latestage preclinical Alzheimer's disease, which does not yet meet the criteria for MCI or prodromal Alzheimer's disease. $^{44}$

The second set of research criteria for Alzheimer's disease $\mathrm{e}^{45}$ were proposed by the NIA-AA working group in 2018. Consistent with the 2014 IWG criteria, ${ }^{42}$ Alzheimer's disease was defined by the presence of amyloid and tau pathology, which can be identified by biomarkers. A modified biomarker classification system, referred to as the amyloid $\beta$ marker, tau pathology, and neurodegeneration system (also known as the ATN system) that was first proposed in 2016, has been adopted on the basis of these criteria. ${ }^{46}$ According to this system, amyloid markers refer to the concentration of amyloid $\beta$ peptide $1-42$ or the amyloid $\beta$ peptide $1-42$ to amyloid $\beta$ peptide $1-40$ concentration ratio in the CSF and amyloid $\beta$ PET. Tau pathology is indicated by the concentration of phosphorylated tau protein in the CSF or tau protein PET. Neurodegeneration is indicated by the concentration of total tau protein in the CSF, atrophy markers by MRI scan, and hypometabolism by 18 F-fluorodeoxyglucose-PET scan. Positive results for the amyloid marker and tau pathology, with or without evidence for neurodegeneration, indicate Alzheimer's disease. ${ }^{46}$

According to the NIA-AA criteria, ${ }^{45}$ amyloid markerpositive and tau pathology-negative results are considered to indicate Alzheimer's disease-associated pathological change. Alzheimer's disease and Alzheimer's diseaseassociated pathological changes together define the Alzheimer's disease continuum. Amyloid marker-negative and tau pathology-positive results indicate an absence of the Alzheimer's disease continuum. ${ }^{45}$

According to the NIA-AA classification system, ${ }^{45}$ the symptomatology of Alzheimer's disease is classified into six numeric stages that reflect the increasing severity of the disease, starting from the initial preclinical phase, where individuals do not experience symptoms (also known as the cognitively unimpaired phase), to MCI, mild dementia, moderate dementia, and finally to severe dementia (figure 2). Additionally, subtle cognitive changes (including those indicated by subjective experiences of cognitive decline) that occur at the late stage of the preclinical, cognitively unimpaired phase of the disease, are classified as the transitional stage (stage 2) of the Alzheimer's disease continuum (figure 2). ${ }^{45}$ Importantly, these NIA-AA criteria ${ }^{45}$ are explicitly proposed for research purposes and are not recommended for use in clinical practice until they have been validated in a clinical setting and their value has been established. A priority of ongoing research is therefore to determine whether the concept and criteria of SCD are useful as a marker for biomarkerbased identification of individuals in stage 2 of the Alzheimer's disease continuum (in which individuals do not yet have objective cognitive decline), and, if so, to identify the risk that these individuals will progress to MCI or dementia. 


\section{Biomarker studies}

Several studies investigating biomarkers of Alzheimer's disease in individuals with SCD, including studies in memory clinics or those from longitudinal populationbased research cohorts, for which cognitive concerns were assessed with questionnaires, have found a link between SCD and Alzheimer's disease. Previous studies ${ }^{47,48,49}$ that measured biomarkers of Alzheimer's disease in individuals with SCD who seek medical help, found that the number of individuals considered to be in the preclinical stage of the Alzheimer's disease continuum (amyloid marker-positive and tau pathology-negative, or amyloid marker-positive and tau pathology-positive) ranged from approximately $7 \%$ to $40 \%$. In some studies, ${ }^{26,50,51}$ amyloid and tau protein PET imaging investigations in cohorts of ageing individuals (>60 years of age) who were cognitively unimpaired showed a quantitative association between the extent of either amyloid or tau pathology with the severity of cognitive concerns experienced by individuals, as measured by use of quantitative scales. These findings are consistent with post-mortem studies, ${ }^{52,53}$ which provided a link between reported subjective decline of cognitive function and subsequent Alzheimer's disease pathology at brain autopsy.

Some of the SCD plus features that have been found to be associated with an increased risk of cognitive decline, have also been studied in preclinical Alzheimer's disease biomarker research. Among individuals who are cognitively unimpaired but have subjective cognitive concerns, those who express particular concerns or worries about their cognitive decline are more likely to be in the preclinical stage of the Alzheimer's disease than individuals with subjective cognitive decline who do not explicitly express concerns about their cognitive function. ${ }^{13,26,54}$ Preclinical Alzheimer's disease is more frequent in individuals with SCD who seek medical help than in research participants with SCD who are recruited as volunteers from the general population..$^{6,29,47,55}$ Similar to individuals who are not cognitively impaired, individuals with subjective cognitive concerns who harbour the major genetic risk factor for Alzheimer's disease (apolipoprotein E4), are at an increased risk of being at the preclinical stage of Alzheimer's disease than individuals with subjective cognitive concerns who do not carry the diseaseassociated apolipoprotein E4 gene. ${ }^{56,57}$ As such, apolipoprotein E4 genotyping can be useful when a group of individuals with SCD who are likely to have preclinical Alzheimer's disease need to be sampled-eg, for a clinical trial.

Longitudinal studies ${ }^{48,49}$ have shown that cognitively unimpaired individuals who have preclinical Alzheimer's disease and who report subjective feelings of cognitive decline, have a $40-62 \%$ risk of progressing either to $\mathrm{MCI}$ or dementia within 3 years. One study ${ }^{58}$ reported that $19 \%$ of these individuals progress to dementia after 6 years. The same study did not report the proportion of patients who progressed to MCI. Cognitively unimpaired

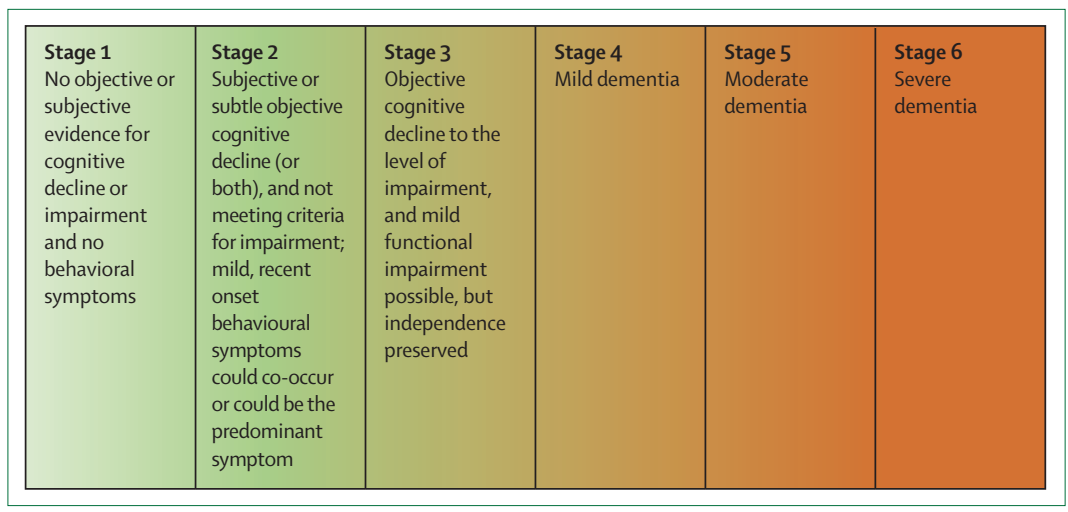

Figure 2: Symptomatic stages of Alzheimer's disease according the NIA-AA research framework The schematic shows the symptomatic stages of disease in Alzheimer's disease according to the NIA-AA research framework $^{45}$ of Alzheimer's disease. The stages apply only to individuals who are in the Alzheimer's disease continuum, which is defined by biomarker evidence of amyloid pathology with or without tau pathology, and is irrespective of the status of neurodegeneration. The colour scheme indicates the continuous progression of cognitive impairment in an individual, from no objective cognitive impairment (light green) to severe objective cognitive impairment (dark red). According to the framework, there are six stages of symptomatic manifestation of Alzheimer's disease. Stage 1 is the fully asymptomatic disease stage. Stage 2, also known as the transitional stage, includes individuals showing the first subtle signs of Alzheimer's disease, manifesting as a combination of one or more of the following symptoms: subjective cognitive decline, subtle objective decline, or mild behavioura symptoms. Stage 1 and stage 2 are classified as preclinical Alzheimer's disease, according to the 2011 NIA-AA criteria. ${ }^{44}$ Stage 3 reflects, in principle, mild cognitive impairment, whereas stages 4 reflects mild dementia, stage 5 reflects moderate dementia, and stage 6 reflects severe dementia. NIA-AA=National Institute of Aging-Alzheimer's Association.

individuals who have preclinical Alzheimer's disease and more severe subjective concerns about their cognition (as measured by use of quantitative scales for subjective concerns) show a faster decline in objective cognition than those with less severe subjective concerns. ${ }^{54,59}$.

Studies on cognitive decline that have recruited volunteers through advertisements show contradictory findings. In one such study that used partial advertisementbased recruitment of individuals, ${ }^{60}$ cognitive decline after 30 months of follow-up was not observed in individuals who were amyloid-positive (by PET scan) and who had subjective cognitive concerns. However, in a latent class growth modelling analysis of results from the same study, ${ }^{61}$ individuals who were amyloid-positive showed a lower performing cognitive trajectory than individuals who were amyloid-negative.

Overall, we consider that the accumulating data provide supporting evidence that SCD corresponds to stage 2 of the NIA-AA Alzheimer's disease framework, and represents the late preclinical phase of Alzheimer's disease. $^{45}$ Of note, results on the predictive value of biomarkers for clinical progression of MCI or dementia are on a group level (ie, there is insufficient data to establish whether biomarkers can predict clinical progression of cognitive decline at an individual level), which hinders the use of biomarkers to predict cognitive decline in individuals with SCD who visit a memory clinic at present.

Other studies have investigated markers of neurodegeneration in individuals with SCD. In particular, crosssectional studies of individuals with SCD from memory 
Search strategy and selection criteria

References for this Personal View were identified by searching PubMed for articles published in English up to July 2019 (without a starting date) and from the references of selected articles. The following search terms were used: "subjective cognitive decline", "SCD", "subjective cognitive impairment", "subjective memory impairment", "cognitive complaint", "cognitive concerns", "memory complaint", and "memory concerns". Full documentation of all search results has not been included in this Personal View. The reference list was generated based on relevance to the topic of this Personal View.

clinics $^{62-64}$ have shown that these individuals have small reductions in the volume of brain regions that typically show loss of volume in the early stages of Alzheimer's disease (eg, the medial temporal lobe) when compared with control groups of individuals without SCD. Some studies $^{63,65}$ have identified Alzheimer's disease-associated patterns of glucose hypometabolism in individuals with SCD, whereas other studies ${ }^{56}$ have not.

There are several major limitations of the research on the association between biomarkers of Alzheimer's disease and SCD. First, only a small number of studies have investigated this association, and these studies involve different research settings and operationalisations of SCD. Second, no longitudinal studies have monitored changes in biomarkers of Alzheimer's disease in cognitively unimpaired individuals from the onset and throughout the subsequent course of SCD and objective cognitive decline. Finally, more studies that focus on markers other than amyloid and tau protein in individuals with SCD are needed, as these markers do not account for SCD in the context of other neurodegenerative diseases.

\section{Conclusions and future directions}

Clinicians are faced with an increasing number of individuals who seek medical help because they experience a subjective decline in cognitive function. There is evidence that individuals with SCD are at a greater risk of future cognitive decline and dementia than individuals who are cognitively unimpaired and do not have subjective cognitive decline. SCD could also be the first symptom of incipient neurodegenerative disease. However, several medical conditions can cause SCD, and research suggests that SCD is not an indicator of future cognitive decline for most individuals. We propose that clinical decisions for individuals who actively seek medical help should be tailored according to their indidivual needs. We also propose that advice about maintenance of brain health should be given to individuals with SCD. There is a strong interest in exploring the potential role of SCD as an early sign of neurodegenerative disorders. This research might become relevant if SCD could be combined with biomarker-based neurodegenerative disease detection and early interventions in the future.

\section{Contributors}

FJ proposed the concept, searched the literature, and drafted the initial manuscript. All remaining authors discussed the selection of literature and contributed to the editing of the manuscript. All authors approved the final manuscript.

\section{Declaration of interests}

We declare no financial relationships directly related to this work. The following grants focus on the topic and results of this article, or support the author's related research: DZNE-DELCODE, BN012 (FJ); Medit-Ageing EU H2020, call PHC22 (FJ, JLM); AMYPAD EU-EFPIA IMI 2, grant 115952 (FJ, JLM); MOPEAD EU-EFPIA IMI 2, grant 115985 (FJ, ORG); EU JPND, Germany (BMBF grant 01ED1508 [FJ], the Netherlands [WMvdF]); SCIENCe project, Gieskes Strijbis Fonds (WMvdF); WMvdF holds the Pasman chair; Zon-MW Off Road, grant 451001010 (SAMS); Key Project, grant 61633018 (NSFC; YH); IH/NIA 1R01AG058825-01A1 (REA); National Institute on Aging, grants R01 AG019771, P30 AG010133, and U01 AG024904 (AJS).

The following financial relationships outside of the presented work include the following: personal fees for advice from Eli Lilly, Biogen, MSD Roche, Nutricia, and Janssen-Cilag, and grant support from Eli Lilly (FJ); grant support from Gieskes-Strijbis fonds, ZonMW, CVON, Pasman stichting, Biogen MA, Combinostics, NWO, EU-FP7, Piramal Neuroimaging, and Janssen-Stellar (WMvdF); personal fees for advice from Roche Diagnostics, Eisai, Oryzon, Roche, Biogen, and Novartis, and grant support from General Electric (JLM); fees for advice from Neurotrack and Eli Lilly (DMR); grant support from NIA (AJS); grant support from ZonMW Off Road (SAMS); fees for advice from Neurotrack and Eli Lilly (CMS); and REA, RFB, YH, LR, ORG, SW, and MW report no disclosures.

\section{Acknowledgments}

This article was produced by representatives of the SCD-I, which is an international working group that aims to conceptualise SCD, provide guidance papers, and promote SCD-related research.

\section{References}

1 Winblad B, Amouyel P, Andrieu S, et al. Defeating Alzheimer's disease and other dementias: a priority for European science and society. Lancet Neurol 2016; 15: 455-532.

2 McKhann GM, Knopman DS, Chertkow H, et al. The diagnosis of dementia due to Alzheimer's disease: recommendations from the National Institute on Aging-Alzheimer's Association workgroups on diagnostic guidelines for Alzheimer's disease. Alzheimers Dement 2011; 7: 263-69.

3 Petersen RC. Mild cognitive impairment as a diagnostic entity. J Intern Med 2004; 256: 183-94.

4 Jessen F, Amariglio RE, Van Boxtel M, et al. A conceptual framework for research on subjective cognitive decline in preclinical Alzheimer's disease. Alzheimers Dement 2014; 10: 844-52.

5 Mitchell A, Beaumont H, Ferguson D, Yadegarfar M, Stubbs B. Risk of dementia and mild cognitive impairment in older people with subjective memory complaints: meta-analysis. Acta Psychiatr Scand 2014; 130: 439-51.

6 Slot RER, Sikkes SAM, Berkhof J, et al. Subjective cognitive decline and rates of incident Alzheimer's disease and non-Alzheimer's disease dementia. Alzheimers Dement 2019; 15: 456-76.

7 Reisberg B, Ferris SH, de Leon MJ, Crook T. The global deterioration scale for assessment of primary degenerative dementia. Am J Psychiatry 1982; 139: 1136-39.

8 Rabin LA, Smart CM, Crane PK, et al. Subjective cognitive decline in older adults: an overview of self-report measures used across 19 international research studies. J Alzheimers Dis 2015; 48 (suppl 1): S63-86.

9 Amariglio RE, Mormino EC, Pietras AC, et al. Subjective cognitive concerns, amyloid- $\beta$, and neurodegeneration in clinically normal elderly. Neurology 2015; 85: 56-62.

10 Rattanabannakit C, Risacher SL, Gao S, et al. The cognitive change index as a measure of self and informant perception of cognitive decline: relation to neuropsychological tests. J Alzheimers Dis 2016; 51: 1145-55.

11 Tomaszewski Farias S, Mungas D, Harvey DJ, Simmons A, Reed BR, Decarli C. The measurement of everyday cognition: development and validation of a short form of the everyday cognition scales. Alzheimers Dement 2011; 7: 593-601. 
12 Rami L, Mollica MA, García-Sanchez C, et al. The subjective cognitive decline questionnaire (SCD-Q): a validation study. J Alzheimers Dis 2014; 41: 453-66.

13 Miebach L, Wolfsgruber S, Polcher A, et al. Which features of subjective cognitive decline are related to amyloid pathology? Findings from the DELCODE study. Alzheimers Res Ther 2019; 11: 66

14 Jessen F, Wiese B, Bachmann C, et al. Prediction of dementia by subjective memory impairment effects of severity and temporal association with cognitive impairment. Arch Gen Psychiatry 2010; 67: 414-22

15 Albert MS, DeKosky ST, Dickson D, et al. The diagnosis of mild cognitive impairment due to Alzheimer's disease: recommendations from the National Institute on Aging-Alzheimer's Association workgroups on diagnostic guidelines for Alzheimer's disease. Alzheimers Dement 2011 7: 270-79.

16 Tsoi KKF, Chan JYC, Hirai HW, et al. Recall tests are effective to detect mild cognitive impairment: a systematic review and meta-analysis of 108 diagnostic studies. J Am Med Dir Assoc 2017; 18: 807.e17-29.

17 Nelson AP, O'Connor MG. Mild cognitive impairment: a neuropsychological perspective. CNS Spectr 2008; 13: 56-64.

18 Bondi MW, Edmonds EC, Jak AJ, et al. Neuropsychological criteria for mild cognitive impairment improves diagnostic precision, biomarker associations, and progression rates. J Alzheimers Dis 2014; 42: 275-89.

19 Molinuevo JL, Rabin LA, Amariglio R, et al. Implementation of subjective cognitive decline criteria in research studies. Alzheimers Dement 2017; 13: 296-311.

20 Van Der Flier WM, Scheltens P. Amsterdam dementia cohort: performing research to optimize care. J Alzheimers Dis 2018 62: 1091-111.

21 Harada CN, Natelson Love MC, Triebel KL. Normal cognitive aging. Clin Geriatr Med 2013; 29: 737-52.

22 Hoogendam YY, Hofman A, Van Der Geest JN, Van Der Lugt A Ikram MA. Patterns of cognitive function in aging: the Rotterdam study. Eur J Epidemiol 2014; 29: 133-40.

23 van Harten AC, Mielke MM, Swenson-Dravis DM, et al. Subjective cognitive decline and risk of MCI. Neurology 2018; 91: e300-12.

24 Amieva H, Le Goff M, Millet X, et al. Prodromal Alzheimer's disease: successive emergence of the clinical symptoms. Ann Neuro 2008; 64: 492-98

25 Verlinden VIA, Van Der Geest JN, De Bruijn RFAG, Hofman A Koudstaal PJ, Ikram MA. Trajectories of decline in cognition and daily functioning in preclinical dementia. Alzheimers Dement 2016 12: $144-53$

26 Verfaillie SCJ, Timmers T, Slot RER, et al. Amyloid- $\beta$ load is related to worries, but not to severity of cognitive complaints in individuals with subjective cognitive decline: the SCIENCe project. Front Aging Neurosci 2019; 11: 7.

27 Roehr S, Villringer A, Angermeyer MC, Luck T, Riedel-Heller SG. Outcomes of stable and unstable patterns of subjective cognitive decline - results from the Leipzig longitudinal study of the aged (LEILA75+). BMC Geriatr 2016; 16: 180

28 Wolfsgruber S, Kleineidam L, Wagner M, et al. Differential risk of incident Alzheimer's disease dementia in stable versus unstable patterns of subjective cognitive decline. J Alzheimers Dis 2016; 54: $1135-46$.

29 Snitz BE, Wang T, Cloonan YK, et al. Risk of progression from subjective cognitive decline to mild cognitive impairment: the role of study setting. Alzheimers Dement 2018; 14: 734-42.

30 Valech N, Mollica MA, Olives J, et al. Informants' perception of subjective cognitive decline helps to discriminate preclinical Alzheimer's disease from normal aging. I Alzheimers Dis 2015; 48 (suppl 1): S87-98.

31 Caselli RJ, Chen K, Locke DEC, et al. Subjective cognitive decline: self and informant comparisons. Alzheimers Dement 2014; 10: 93-98.

32 Nicholas CR, Dowling NM, Racine AM, et al. Longitudinal assessment of self- and informant-subjective cognitive complaints in a sample of healthy late-middle aged adults enriched with a family history of Alzheimer's disease. J Int Neuropsychol Soc 2017; 23: $617-26$
33 Valech N, Tort-Merino A, Coll-Padrós N, et al. Executive and language subjective cognitive decline complaints discriminate preclinical Alzheimer's disease from normal aging. J Alzheimers Dis 2018; 61: 689-703.

34 La Joie R, Perrotin A, Egret S, et al. Qualitative and quantitative assessment of self-reported cognitive difficulties in nondemented elders: association with medical help seeking, cognitive deficits, and $\beta$-amyloid imaging. Alzheimers Dement (Amst) 2016; 5: $23-34$

35 Wu Q. Subjective cognitive impairment of older adults: a comparison between the US and China. Int J Methods Psychiatr Res 2016; 25: 68-75.

36 Jackson JD, Rentz DM, Aghjayan SL, et al. Subjective cognitive concerns are associated with objective memory performance in Caucasian but not African-American persons. Age Ageing 2017; 46: 988-93.

37 Garcia-Ptacek S, Cavallin L, Kåreholt I, et al. Subjective cognitive impairment subjects in our clinical practice. Dement Geriatr Cogn Dis Extra 2014; 4: 419-30.

38 Comijs HC, Deeg DJH, Dik MG, Twisk JWR, Jonker C. Memory complaints; the association with psycho-affective and health problems and the role of personality characteristics. A 6-year follow-up study. J Affect Disord 2002; 72: 157-65.

39 Hill NL, Mogle J, Wion R, et al. Subjective cognitive impairment and affective symptoms: a systematic review. Gerontologist 2016; 56: e109-27.

40 Livingston G, Sommerlad A, Orgeta V, et al. Dementia prevention, intervention, and care. Lancet 2017; 390: 2673-734.

41 Wesselman LMP, Schild A-K, Coll-Padros N, et al. Wishes and preferences for an online lifestyle program for brain health-a mixed methods study. Alzheimers Dement (NY) 2018; 4: 141-49.

42 Dubois B, Feldman HH, Jacova C, et al. Advancing research diagnostic criteria for Alzheimer's disease: the IWG-2 criteria. Lancet Neurol 2014; 13: 614-29.

43 Dubois B, Hampel H, Feldman HH, et al. Preclinical Alzheimer's disease: definition, natural history, and diagnostic criteria. Alzheimers Dement 2016; 12: 292-323.

44 Sperling RA, Aisen PS, Beckett LA, et al. Toward defining the preclinical stages of Alzheimer's disease: recommendations from the National Institute on Aging-Alzheimer's Association workgroups on diagnostic guidelines for Alzheimer's disease. Alzheimers Dement 2011; 7: 280-92.

45 Jack CR, Bennett DA, Blennow K, et al. NIA-AA research framework: toward a biological definition of Alzheimer's disease. Alzheimers Dement 2018; 14: 535-62.

46 Jack CR, Bennett DA, Blennow K, et al. A/T/N: an unbiased descriptive classification scheme for Alzheimer disease biomarkers. Neurology 2016; 87: 539-47.

47 Jessen F, Spottke A, Boecker H, et al. Design and first baseline data of the DZNE multicenter observational study on predementia Alzheimer's disease (DELCODE). Alzheimers Res Ther 2018; 10: 15.

48 van Harten AC, Smits LL, Teunissen CE, et al. Preclinical AD predicts decline in memory and executive functions in subjective complaints. Neurology 2013; 81: 1409-16.

49 Wolfsgruber S, Polcher A, Koppara A, et al. Cerebrospinal fluid biomarkers and clinical progression in patients with subjective cognitive decline and mild cognitive impairment. J Alzheimers Dis 2017; 58: 939-50.

50 Amariglio RE, Becker JA, Carmasin J, et al. Subjective cognitive complaints and amyloid burden in cognitively normal older individuals. Neuropsychologia 2012; 50: 2880-86.

51 Buckley RF, Hanseeuw B, Schultz AP, et al. Region-specific association of subjective cognitive decline with tauopathy independent of global $\beta$-amyloid burden. JAMA Neurol 2017; 74: $1455-63$

52 Arvanitakis Z, Leurgans SE, Fleischman DA, et al. Memory complaints, dementia, and neuropathology in older blacks and whites. Ann Neurol 2018; 83: 718-29.

53 Kryscio RJ, Abner EL, Cooper GE, et al. Self-reported memory complaints: implications from a longitudinal cohort with autopsies Neurology 2014; 83: 1359-65.

54 Amariglio RE, Buckley RF, Mormino EC, et al. Amyloid-associated increases in longitudinal report of subjective cognitive complaints. Alzheimers Dement (NY) 2018; 4: 444-49. 
55 Rodríguez-Gómez O, Abdelnour C, Jessen F, Valero S, Boada M. Influence of sampling and recruitment methods in studies of subjective cognitive decline. J Alzheimers Dis 2015; 48 (suppl 1): S99-107

56 Risacher SL, Kim S, Nho K, et al. APOE effect on Alzheimer's disease biomarkers in older adults with significant memory concern. Alzheimers Dement 2015; 11: 1417-29.

57 Moreno-Grau S, Rodríguez-Gómez O, Sanabria Á, et al. Exploring APOE genotype effects on Alzheimer's disease risk and amyloid $\beta$ burden in individuals with subjective cognitive decline: the FundacioACE healthy brain initiative (FACEHBI) study baseline results. Alzheimers Dement 2018; 14: 634-43.

58 Hessen E, Eckerström M, Nordlund A, et al. subjective cognitive impairment is a predominantly benign condition in memory clinic patients followed for 6 years: the Gothenburg-Oslo MCI study. Dement Geriatr Cogn Dis Extra 2017; 7: 1-14.

59 Vogel JW, Varga Dolezalova M, La Joie R, et al. Subjective cognitive decline and $\beta$-amyloid burden predict cognitive change in healthy elderly. Neurology 2017; 89: 2002-09.

60 Dubois B, Epelbaum S, Nyasse F, et al. Cognitive and neuroimaging features and brain $\beta$-amyloidosis in individuals at risk of Alzheimer's disease (INSIGHT-preAD): a longitudinal observational study. Lancet Neurol 2018; 17: 335-46.
61 Teipel SJ, Cavedo E, Lista S, et al. Effect of Alzheimer's disease risk and protective factors on cognitive trajectories in subjective memory complainers: an INSIGHT-preAD study. Alzheimers Dement 2018; 14: 1126-36.

62 Saykin AJ, Wishart HA, Rabin LA, et al. Older adults with cognitive complaints show brain atrophy similar to that of amnestic MCI. Neurology 2006; 67: 834-42.

63 Scheef L, Spottke A, Daerr M, et al. Glucose metabolism, gray matter structure, and memory decline in subjective memory impairment. Neurology 2012; 79: 1332-39.

64 Perrotin A, La Joie R, de La Sayette V, et al. Subjective cognitive decline in cognitively normal elders from the community or from a memory clinic: differential affective and imaging correlates. Alzheimers Dement 2017; 13: 550-60.

65 Vannini P, Hanseeuw B, Munro CE, et al. Hippocampal hypometabolism in older adults with memory complaints and increased amyloid burden. Neurology 2017; 88: 1759-67.

(C) 2020 Elsevier Ltd. All rights reserved. 